\title{
Platelet Hematocrit Measurement
}

National Cancer Institute

\section{Source}

National Cancer Institute. Platelet Hematocrit Measurement. NCI Thesaurus. Code C100424.

The determination of the amount of platelet hematocrit present in a sample. 